\title{
Coupled equations for transient water flow, heat flow, and deformation in hydrogeological systems
}

\author{
T N NARASIMHAN \\ Department of Materials Science and Engineering, Department of Environmental Science, \\ Policy and Management, 210 Hearst Memorial Mining Building, \\ University of California at Berkeley, Berkeley, Ca 94720-1760, USA. \\ e-mail: tnnarasimhan@lbl.gov
}

\begin{abstract}
Hydrogeological systems are earth systems influenced by water. Their behaviors are governed by interacting processes, including flow of fluids, deformation of porous materials, chemical reactions, and transport of matter and energy. Here, coupling among three of these processes is considered: flow of water, heat, and deformation, each of which is represented by a diffusion-type of partial differential equation. One side of the diffusion-type equation relates to motion of matter or energy, while the other relates to temporal changes of state variables at a given location. The coupling arises from processes that govern motion as well as those that relate to change of state. In this work, attention is devoted to coupling arising from changes in state. Partial derivatives of equations of state constitute the capacitance terms of diffusion-type equations. Of the many partial derivatives that are mathematically possible in physical systems characterized by several variables, only a few are physically significant. Because the state variables are related to each other through an equation of state, the partial derivatives must collectively satisfy a closure criterion. This framework offers a systematic way of developing the coupled set of equations that govern hydrogeological systems involving the flow of water, heat, and deformation. Such systems are described in terms of four variables, and the associated partial derivatives. The physical import of these derivatives are discussed, followed by a description of partial derivatives that are of practical interest. These partial derivatives are then used as the basis to develop a set of coupled equations. A brief discussion is presented on coupled equations from a perspective of energy optimization.
\end{abstract}

\section{Introduction}

Given an appropriate time scale, all earth systems are transient. The evolution of the earth's crust involves interaction of a variety of processes including transport of fluids and energy, deformation, chemical reactions, and transport of chemical constituents. The diffusion equation, originally proposed by Fourier early in the nineteenth century, has grown to constitute the mathematical framework in which most transient transport processes of energy and matter are described. Consequently, coupled processes that describe the evolution of hydrogeological systems are expressed as a set of partial differential equations that are tied together through physical attributes that overlap among the different processes. This work is concerned with systematically developing a set of coupled equations relevant to hydrogeological systems involving flow water and heat, accompanied by deformation of porous geological media.

The diffusion-type equation is of the form,

$$
\nabla \cdot K \nabla T=c \frac{\partial T}{\partial t}
$$

where, $\nabla$ is a spatial gradient operator, $K$ is conductivity (a parameter), $T$ is an intensive quantity

Keywords. Coupled processes; hydrogeological systems; heat and mass transport.

J. Earth Syst. Sci. 115, No. 2, April 2006, pp. 219-228

(C) Printed in India. 
(dependent variable), $c$ is specific volumetric capacity (a parameter), and $t$ is time. Physically, the equation represents the conservation, per unit volume, of an extensive quantity over an infinitesimal volume element centered at a point in the flow domain. Mathematically, $T$ is a continuous function, twice differentiable in space and once in time. The conductivity parameter relates the flux of the extensive quantity to the spatial gradient of $T$ over an interval of time during which the gradient remains unchanged. The capacity parameter relates, at a given location, the change in the amount of the extensive quantity in an elemental volume to the average change in $T$ over the volume, assuming that the two quantities are in equilibrium.

The attributes that are responsible for coupling the equations may be related to either the equations of motion or to the equations of state. In the present work, we devote attention to coupling attributes that arise from the equation of

$$
\left[\begin{array}{cc}
1 & \left(\frac{\partial p}{\partial M_{w}}\right)_{\sigma, T} \\
\left(\frac{\partial M_{w}}{\partial p}\right)_{\sigma, T} & 1 \\
\left(\frac{\partial \sigma}{\partial p}\right)_{M_{w, T}} & \left(\frac{\partial \sigma}{\partial M_{w}}\right)_{p, T} \\
\left(\frac{\partial T}{\partial p}\right)_{M_{w}, \sigma} & \left(\frac{\partial T}{M_{w}}\right)_{p, \sigma}
\end{array}\right.
$$

state, as inherent in the partial derivatives. Coupling attributes related to the equations of motion arise as consequence of Onsager's reciprocal relationships, according to which, motion of water may be impelled by a temperature gradient, or motion of heat may be caused by a pressure gradient. These phenomena are beyond the scope of the present work.

\section{A four-variable system}

Consider a water-saturated non-steady flow system, in combination with transient heat flow and three-dimensional deformation. The goal is to develop equations governing the evolution of this system in time, in response to interactions among the relevant processes. The porous medium is elastic, subject to small deformations.
In general, this system is characterized by five observable state variables. These are, $M_{w}$ (mass of water), $p$ (water pressure), $\sigma$ (external stress acting on the solid phases), $T$ (temperature), and, $E$ (heat energy). However, a simplifying assumption is made that $T$ and $E$ are uniquely related to each other. That is, change in temperature can occur only due to change in the amount of heat energy, although change in temperature can cause other state variables to change. As a consequence, attention is restricted to temperature, and energy is ignored in defining the state variables. Thus, the number of state variables reduces to four. System behavior is quantified only through these four variables. The system is described in terms of an equation of state of the form,

$$
F\left(p, M_{w}, \sigma, T\right)=0 .
$$

This system permits 12 partial derivatives, which occupy off-diagonal positions in the following matrix.

$$
\left.\begin{array}{cc}
\left(\frac{\partial p}{\partial \sigma}\right)_{M_{w}, T} & \left(\frac{\partial p}{\partial T}\right)_{M_{w, \sigma}} \\
\left(\frac{\partial M_{w}}{\partial \sigma}\right)_{p, T} & \left(\frac{\partial M_{w}}{\partial T}\right)_{p, \sigma} \\
1 & \left(\frac{\partial \sigma}{\partial T}\right)_{p, M_{w}} \\
\left(\frac{\partial T}{\partial \sigma}\right)_{p, M_{w}} & 1
\end{array}\right]
$$

If the mutual relations between each pair of state variable were to be fully reversible, then each term below the leading diagonal will be the reciprocal of the corresponding term above that diagonal. In this sense, the matrix is phenomenologically symmetric. However, it will be seen later that not all of the partial derivatives are physically meaningful. In actuality, therefore, the symmetry is superficial.

Assume that at an initial time $t_{0}$, these variables take on values of $p^{0}, M_{w}^{0}, \sigma_{i j}^{0}$ and $T^{0}$ at a given location. Let the total changes in these variables over a small time interval $\Delta t$ be $d p, d M_{w}, d \sigma$ and $d T$. Then, between $t_{0}$ and $t_{0}+\Delta t$ the system would have evolved to,

$$
\begin{aligned}
p\left(t_{0}+\Delta t\right) & =p^{0}+d p, \\
M_{w}\left(t_{0}+\Delta t\right) & =M_{w}^{0}+d M_{w}, \\
\sigma\left(t_{0}+\Delta t\right) & =\sigma^{0}+d \sigma,
\end{aligned}
$$


and,

$$
T\left(t_{0}+\Delta t\right)=T^{0}+d T
$$

In order to describe the evolution of the system, therefore, governing equations are to be derived for each of the four total differentials. But, we recognize that each of these total differentials is a sum of the component partial differentials as follows, assuming that temperature can change only due to the change in sensible heat, neglecting possible effects of heat generation due to friction.

$$
\begin{aligned}
d p & =(\partial p)_{\sigma, T}+(\partial p)_{M_{w}, T}+(\partial p)_{M_{w}, \sigma}, \\
d M_{w} & =\left(\partial M_{w}\right)_{\sigma, T}+\left(\partial M_{w}\right)_{p, T}+\left(\partial M_{w}\right)_{p, \sigma}, \\
d \sigma & =(\partial \sigma)_{M_{w}, T}+(\partial \sigma)_{p, T}+(\partial \sigma)_{p, M_{w}}, \\
d T & =(\partial T)_{p, M_{w}, \sigma} .
\end{aligned}
$$

In the above, the subscripts denote the variables which are held fixed. In principle, one equation will have to be derived for each of the partial derivatives so that all the four total differentials can be evaluated. However, physically, some of the partials will not be relevant and hence, these partials can be ignored. The next step is to examine the physical significance of each partial derivative.

\section{Physical significance of partial derivatives}

In the following physical description, the convention followed is that the numerator is the effect, caused by the denominator.

\section{$\left(\partial p / \partial M_{w}\right)_{\sigma, T}$ Constant-stress drainage: Change in pressure due to change in fluid mass. This differential underlies the equations of tran- sient fluid flow widely used in the fields of hydrogeology, soil physics, soil mechanics and petroleum engineering.}

$(\partial p / \partial \sigma)_{M_{w}, T}$ Undrained loading: Change in pressure at constant temperature due to change in external stress, without water draining out. Pressure changes instantaneously in response to stress change. This condition arises in the responses of groundwater systems to earth tides, barometric changes, ocean tides and seismic waves.

$(\partial p / \partial T)_{\sigma, M_{w}}$ Thermal pressurisation: Change in pressure caused by change in temperature, under undrained conditions, with constant external stress. Pressure changes instantaneously in response to temperature change.

$\left(\partial M_{w} / \partial p\right)_{\sigma, T}$ Constant-stress drainage: Reciprocal of $(\partial p)_{\sigma, T}$. Analogous to heat capacity. Change in mass of water in an elemental volume accompanying a unit change in fluid pressure. May be called hydraulic capacity by analogy with heat capacity.

$\left(\partial M_{w} / \partial \sigma\right)_{p, T}$ Drained loading: Change in quantity of water due to change in external stress at constant fluid pressure and constant temperature. Ideally, this partial derivative equals the volume change experienced by the dry porous medium subjected to the same external stress change, multiplied by the density of water.

$\left(\partial M_{w} / \partial T\right)_{\sigma, p}$ Drained heating: Drainage of water induced by heating when external stress and fluid pressure are not allowed to change. At constant external stress and water pressure, the pore volume remains constant, and the thermally expanded water is drained.

$(\partial \sigma / \partial p)_{M_{w}, T} \quad$ Superficially, this is a reciprocal of undrained loading, $(\partial p)_{M_{w}, T}$. However, it is hard to visualize a physical situation in which one can manipulate water pressure inside a porous material without altering the mass of water contained or the temperature, so that external stress can be changed. Therefore, this partial derivative is not of physical interest.

$\left(\partial \sigma / M_{w}\right)_{p, T} \quad$ Stress relaxation: Relaxation of external stress consequent to injection of water at constant pressure under isothermal conditions. Although physically possible in a laboratory with servo-controlled apparatus, this process is not likely to occur under field geological conditions, where $\sigma$ is invariably determined by tectonic causes or by applied boundary loads. Therefore, this differential is not of practical interest.

$(\partial \sigma / \partial T)_{M_{w}, p} \quad$ Thermal stressing: Undrained heating, with the volume allowed deform freely in all directions in such a way that there is no pressure 
Table 1. Summary of partial derivatives and their significance.

\begin{tabular}{|c|c|c|}
\hline $\begin{array}{l}\text { Matrix } \\
\text { index }\end{array}$ & Derivative & Physical significance \\
\hline 1,2 & $\left(\partial p / \partial M_{w}\right)_{\sigma, T}$ & Constant-stress drainage \\
\hline 1,3 & $(\partial p / \partial \sigma)_{M_{w}, T}$ & Undrained loading \\
\hline 1,4 & $(\partial p / \partial T)_{\sigma, M_{w}}$ & Thermal pressurisation \\
\hline 2,1 & $\left(\partial M_{w} / \partial p\right)_{\sigma, T}$ & Constant-stress drainage. Hydraulic capacitance \\
\hline 2,3 & $\left(\partial M_{w} / \partial \sigma\right)_{p, T}$ & Drained loading \\
\hline 2,4 & $\left(\partial M_{w} / \partial T\right)_{\sigma, p}$ & Drained heating \\
\hline 3,1 & $(\partial \sigma / \partial p)_{M_{w}, T}$ & Physically not possible to change stress by manipulating pressure \\
\hline 3,2 & $\left(\partial \sigma / \partial M_{w}\right)_{p, T}$ & Stress relaxation. Physically not realistic in earth systems \\
\hline 3,4 & $(\partial \sigma / \partial T)_{p, M_{w}}$ & Thermal stressing. Physically not realistic in earth systems \\
\hline 4,1 & $(\partial T / \partial p)_{M_{w}, \sigma}$ & Physically not realistic in earth systems \\
\hline 4,2 & $\left(\partial T / \partial M_{w}\right)_{p, \sigma}$ & Physically not realistic in earth systems \\
\hline 4,3 & $(\partial T / \partial \sigma)_{p, M_{w i}}$ & Physically not realistic in earth systems \\
\hline
\end{tabular}

Note: 1. Physically relevant partial derivatives are shown in bold face.

2. In describing the partial derivatives, the convention used is that the numerator is the effect, and the denominator is the cause. Thus, $\left(\partial p / \partial M_{w}\right)_{\sigma, T}$ implies pressure change caused by change in mass of water.

build up. To accommodate thermal expansion, external stress will have to relax. This process, just as stress relaxation, is not of practical interest.

$(\partial T)_{p, M_{w}, T} \quad$ Because $T$ is stipulated to be solely a function only of heat energy, the differential for $T$ is a total differential, $d T$. In other words, the three partial derivatives on the bottom row of the matrix above are of no physical interest in earth systems.

The nature and relevance of the twelve partial derivatives are summarized in table 1. As can be seen from the table, the last six of the twelve partial derivatives are physically unrealistic in earth systems. Of the remaining six, two, namely, drained loading $\left(\partial M_{w} / \partial \sigma\right)_{p, T}$ and drained heating $\left(\partial M_{w} / \partial T\right)_{\sigma, p}$ require time for equilibration because a finite time is needed for water to drain. In practice, these two derivatives can be treated as serial combinations of an instantaneous process followed by a drainage process. Thus, $\left(\partial M_{w} / \partial \sigma\right)_{p, T}$ can be treated as being equivalent to a sequential application of $(\partial p / \partial \sigma)_{M_{w}, T}$, which causes an instantaneous change in pressure, followed by time-dependent drainage through applying $\left(\partial M_{w} / \partial p\right)_{\sigma, T}$. Similarly, $\left(\partial M_{w} / \partial T\right)_{p, \sigma}$ can be treated as being equivalent to a sequential application of $(\partial p / \partial T)_{M_{w}, \sigma}$, which causes an instantaneous change in pressure, followed by time-dependent drainage through applying $\left(\partial M_{w} / \partial p\right)_{\sigma, T}$. Of the remaining four, $\left(\partial M_{w} / \partial p\right)_{\sigma, T}$ and $\left(\partial p / \partial M_{w}\right)_{\sigma, T}$ are reciprocals of each other.
Thus, for developing the coupled equations, only the following three derivatives need consideration.
$\left(\partial M_{w} / \partial p\right)_{\sigma, T} \quad$ Constant-stress drainage
$(\partial p / \partial \sigma)_{M_{w}, T} \quad$ Undrained loading, and
$(\partial p / \partial T)_{M_{w}, \sigma}$ Thermal pressurisation.

Note that of these three partial derivatives, only the first, constant-stress drainage, involves time. The other two, undrained loading and thermal pressurisation represent instantaneous changes of pressure induced by the changes in external stress and temperature respectively.

\section{Expressions for the partial derivatives}

\section{$4.1\left(\partial M_{w} / \partial p\right)_{\sigma, T}$, constant-stress drainage}

Under isothermal conditions, when water is allowed to move freely into and out of a deformable material, change in the mass of water stored occurs due to a change in the pore volume and a simultaneous change in the density of the fluid. The change in the mass of the water in the material per unit change in fluid pressure is the hydraulic capacity of the material, given by,

$$
\left(\frac{\partial M_{w}}{\partial p}\right)_{\sigma, T}=V_{B} \rho\left[\zeta \alpha_{I}+n \beta\right],
$$

where, $V_{B}$ is the bulk volume of the elemental volume, $\rho$ is density of water, $\alpha_{I}$ is the volumetric compressibility of the porous medium, $n$ is porosity, $\beta$ is compressibility of the fluid, and, $\zeta$ is a 
coefficient which relates change in water pressure and change in skeletal stresses. Effective stress is the portion of the external stress that is borne by the porous skeleton when the pores are saturated with water. The volumetric compressibility of the porous material, $\alpha_{I}$ is defined in terms of change in mean principal effective stress, $\sigma_{M}^{\prime}$ as,

$$
\alpha_{I}=-\frac{\partial n}{\partial \sigma_{M}^{\prime}}=-\frac{\partial \varepsilon_{v}}{\partial \sigma_{M}^{\prime}},
$$

where $\epsilon_{v}$ is volumetric strain. Extending Terzaghi's well-known effective stress law to three dimensions (Terzaghi 1923), mean principal stress, $\sigma_{M}^{\prime}$, is defined as,

$$
\sigma_{M}^{\prime}=\frac{\sigma_{1}+\sigma_{2}+\sigma_{3}}{3}-\theta p=\sigma_{M}-\theta p
$$

in which $\sigma_{1}, \sigma_{2}$, and $\sigma_{3}$ are the three principal external stresses, and $\theta=\left(1-K / K_{s}\right)$ where $K$ is the bulk modulus of the porous medium and $K_{s}$ is the bulk modulus of the solid grains. The bulk modulus of the porous medium, $K$, is the reciprocal of the invariant compressibility, $\alpha_{I}$. Except when dealing with deep-earth processes, it is customary to consider the bulk modulus of the solids to be far larger than that of the porous medium and hence, set $\theta=1$ in equation (7). Thus, under conditions of constant external stress, the change in mean principal effective stress is treated as equal to the change in the fluid pressure times $(-1)$. Therefore, it is logical to define hydraulic capacity in terms of $\alpha_{I}$. Defined thus, $\alpha_{I}$ is independent of boundary conditions in an elastic material, and is an invariant quantity, and hence the subscript, $I$. In the above, pore volume change is expressed in terms of skeletal stresses, whereas hydraulic capacity is defined in terms of fluid pressure. In order to express pore volume change in terms of fluid pressure, a coefficient is needed to couple skeletal stresses and fluid pressure. Accordingly, the coupling coefficient $\zeta$ is defined as,

$$
\frac{\partial n}{\partial \sigma_{M}^{\prime}} \frac{\partial \sigma_{M}^{\prime}}{\partial p}=\left(-\alpha_{I}\right)(-\zeta)=\alpha_{I} \zeta
$$

Comparison of equation (8) with equation (7) shows that $\zeta=\theta$.

In groundwater hydrology, it is customary to express the constant-stress drainage coefficient in terms of the volume of water drained (rather than mass of water) per unit volume, per unit change in hydraulic head (rather than pressure). This parameter is referred to as specific storage (Hantush 1961), and is expressed as,

$$
S_{s}=\left(\frac{d n}{d \psi}\right)_{\sigma, T}=\rho g\left[\zeta \alpha_{I}+n \beta\right],
$$

where, $\psi=p /(\rho g)$, is pressure head.

\section{$4.2(\partial p / \partial \sigma)_{M_{w}, T}$, undrained loading}

If we assume that volume change occurs only due to changes in normal stresses and is unaffected by shear stresses at constant mass of water and temperature, the result is undrained loading. In this case, the change in the volume of water is equal to the change in pore volume. This constraint, combined with the effecive stress relation leads to,

$$
\left(\frac{\partial p}{\partial \sigma_{M}}\right)_{M_{w}, T}=\frac{\zeta \alpha_{I}}{\zeta \alpha_{I}+n \beta}=B
$$

where $B$ is a coefficient attributed to Skempton (1954).

\section{$4.3(\partial p / \partial T)_{M_{w}, \sigma}$, thermal pressurisation}

For an elemental volume that is subject to a temperature change with free deformation at constant total stress without allowing water to drain out, the change in water volume caused by thermal expansion must be accommodated by an equivalent change in fluid pressure that will cause the necessary change in pore volume. Thus,

$$
\left(\frac{\partial p}{\partial T}\right)_{M_{w}, \sigma}=\frac{n \beta_{T}-\alpha_{T}}{\zeta \alpha_{I}+n \beta},
$$

where $\alpha_{T}$ is the thermal expansivity of the rock defined by,

$$
\alpha_{T}=\left(\frac{d n}{d T}\right)_{M_{w}, \sigma},
$$

and $\beta_{T}$ is the thermal expansivity of water defined by,

$$
\beta_{T}=\frac{1}{V_{w}}\left(\frac{\Delta V_{w}}{\Delta T}\right)_{M_{w}, \sigma},
$$

where the subscript $w$ denotes water.

The expressions given above for constant-stress drainage, undrained loading and thermal pressurisation are adequate for us to develop the various coupled equations of interest. However, for purposes elucidating the closure property, the following additional expressions are given.

\section{$4.4\left(\partial M_{w} / \partial \sigma\right)_{p, T}$, drained loading}

Under drained loading, the density of water remains constant and hence the volume of water 
drained due to the change in external stress must be accommodated entirely by an equal change in pore volume. Also, an increase in external stress is accompanied by a decrease in pore volume and expulsion of water. As a result, this partial derivative is directly related to compressibility $\alpha_{I}$ by,

$$
\left(\frac{\partial M_{w}}{\partial \sigma}\right)_{p, T}=-V_{B} \rho \alpha_{I}
$$

\section{$4.5(\partial \sigma / \partial T)_{p, M_{w}}$, thermal stressing}

For an elemental volume heated with pressure held constant and water prevented from drainage, the change in water volume must be compensated by an equivalent change in external stresses. Thus,

$$
\left(\frac{\partial \sigma_{M}}{\partial T}\right)_{p, M_{w}}=-\frac{n \beta_{T}-\alpha_{T}}{\zeta \alpha_{I}}
$$

\subsection{Closure criterion}

The expressions detailed above can be checked for mutual consistency through a closure criterion which must hold for a well-defined several-variable system. Thus, if $F\left(x_{1}, x_{2}, x_{3}, \ldots, x_{n}\right)=0$ is true for an $n$-variable system, then, one can show that the partial derivatives of this system must satisfy the closure criterion,

$$
\frac{\partial x_{1}}{\partial x_{2}} \times \frac{\partial x_{2}}{\partial x_{3}} \times \frac{\partial x_{3}}{\partial x_{4}} \times \cdots \times \frac{\partial x_{n}}{\partial x_{1}}=(-1)^{n} .
$$

Applying this criterion to the four-variable system on hand yields,

$$
\begin{aligned}
\left(\frac{\partial p}{\partial M_{w}}\right)_{\sigma, T}\left(\frac{\partial M_{w}}{\partial \sigma}\right)_{p, T}\left(\frac{\partial \sigma}{\partial T}\right)_{\sigma, T}\left(\frac{\partial T}{\partial p}\right)_{\sigma, T} \\
=\left(\frac{1}{V_{B} \rho\left[\zeta \alpha_{I}+n \beta\right]}\right)\left(-V_{B} \rho \alpha_{I}\right)\left(\frac{-\left[n \beta_{T}-\alpha_{T}\right]}{\alpha_{I}}\right) \\
\quad \times\left(\frac{\zeta \alpha_{I+n \beta}}{n \beta_{T}-\alpha_{T}}\right)=(-1)^{4}=1
\end{aligned}
$$

thus verifying the mutual consistency of the parameters.

\section{Three basic coupled equations}

\section{$5.1\left(\partial M_{w} / \partial p\right)_{\sigma, T}:$ Constant-stress drainage}

Consider first an isothermal system in which water moves through a geologic material, with external stress remaining constant. When flow is non-steady, a change in the quantity of mass of the water at a given location will lead to a change in pressure at that location. Clearly, this process involves the partial derivative pertaining to constant-stress drainage. Moreover, the change in the mass of water which causes pressure to change is assumed to occur due to the motion of subsurface fluids in and out of an elemental volume, caused by the combined effects of gravity and spatial variations in pressure that existed over the system at the initial time. In turn, gravity and the spatial variations of pressure on the one hand and the quantity of moving water on the other are related to each other by an empirically postulated equation of motion known as Darcy's Law. Thus, in order to formulate the equation governing isothermal, transient flow a liquid in a deforming medium, one starts with,

$$
\begin{aligned}
\left(\frac{\partial M_{w}}{\partial t}\right)_{\sigma, T} & =\left(\frac{\partial M_{w}}{\partial p}\right)_{\sigma, T}\left(\frac{\partial p}{\partial t}\right)_{\sigma, T} \\
& =V_{B} \rho\left(\zeta \alpha_{I}+n \beta\right)\left(\frac{\partial p}{\partial t}\right)_{\sigma, T} .
\end{aligned}
$$

In the above equation, the lefthand side represents the accumulation of liquid due to spatial variations in the flow of the liquid governed by Darcy's Law,

$$
\begin{aligned}
Q & =\text { Volumetric flux } \\
& =\left(-\frac{k}{\mu}[\rho g \nabla z+\nabla p] A\right)_{\sigma, T},
\end{aligned}
$$

where $k$ is intrinsic permeability dependent on pore geometry of the material, $\mu$ is viscosity of water, $g$ is acceleration due to gravity, $z$ is elevation and $A$ is area of cross section. In view of equation (19), we may express equation (18) in the form of a partial differential equation,

$$
\left(\nabla \cdot \rho \frac{k}{\mu}[\rho g \nabla z+\nabla p]\right)_{\sigma, T}=\rho\left[\zeta \alpha_{I}+n \beta\right]\left(\frac{\partial p}{\partial t}\right)_{\sigma, T} .
$$

This equation expresses the conservation of mass of water in units of mass of water. However, it is a long tradition in the earth sciences to express mass conservation in terms of volume of the liquid rather than its mass. Accordingly, dividing both sides by $\rho$ yields,

$$
\left(\nabla \cdot \frac{k}{\mu}[\rho g \nabla z+\nabla p]\right)_{\sigma, T}=\left[\zeta \alpha_{I}+n \beta\right]\left(\frac{\partial p}{\partial t}\right)_{\sigma, T},
$$


which is essentially the form in which the transient flow of oil is expressed in the petroleum engineering literature.

In the fields of groundwater, soil physics and civil engineering, it is customary to use pressure head as the dependent variable rather than pressure because it is practically convenient to measure depth or elevations of water levels in wells and bore holes. Accordingly, equation (21) takes the form,

$$
\begin{aligned}
(\nabla \cdot K \nabla[z+\psi])_{\sigma, T} & =\rho g\left[\zeta \alpha_{I}+n \beta\right]\left(\frac{\partial \psi}{\partial t}\right)_{\sigma, T} \\
& =S_{s}\left(\frac{\partial \psi}{\partial t}\right)_{\sigma, T},
\end{aligned}
$$

where $K=(k \rho g / \mu)$ is hydraulic conductivity, $\psi=p /(\rho g)$ is pressure head and $S_{s}$ is specific storage.

If our interest is restricted to purely understanding water pressure changes over the domain, then, equations (20) to (22) are adequate. In so far as water is concerned, it sees only volumetric strains and the corresponding changes in fluid pressure. The invariant compressibility term in the capacitance expression adequately accounts for the relation between volumetric strain and fluid pressure change. However, to understand the changes in the stress field in addition to changes in water pressure, equations (20) to (22) are insufficient. In this case, it is necessary to give detailed consideration to the three dimensional nature of the deformation process represented by the compressibility parameter $\alpha_{I}$. This entails a coupling between the transient fluid flow equation, and a three dimensional force balance equation. This coupling was first introduced by Biot (1941).

To derive the coupled equation, deformation of the porous medium is separated from water compressibility, leading to,

$$
\left(\nabla \cdot \frac{k}{\mu}[\rho g \nabla z+\nabla p]\right)_{\sigma, T}=\left(\frac{\partial \varepsilon_{v}}{\partial t}\right)_{\sigma, T}+n \beta\left(\frac{\partial p}{\partial t}\right)_{\sigma, T},
$$

where $\epsilon_{v}$ is the volumetric strain, which for small strains, is taken to be equal to the sum of the strains in the three principal directions. That is, $\epsilon_{v}=\epsilon_{1}+\epsilon_{2}+\epsilon_{3}$. Whereas in the conventional forms of the transient flow equation there is but one unknown (water pressure), equation (23) has three additional unknowns, namely, strains in the three principal directions, $\epsilon_{1}, \epsilon_{2}$, and $\epsilon_{3}$. Therefore, three additional equations need to be developed. For this purpose, force balance equations arising from stress-strain behavior of linear elastic materials is invoked. The partial differential equation of force balance, neglecting body forces for convenience is,

$$
\nabla \cdot \sigma_{i j}=0
$$

where $\sigma_{i j}$ is the tensor of external stress. Because the unknowns on hand are strains, and strains are controlled by effective stress (that is, the difference between external stresses and water pressure), it is necessary to express stresses in terms of strains as follows,

$$
\begin{aligned}
\Delta \sigma_{i j} & =2 \lambda E \varepsilon_{i j}+G \delta_{i j} \delta_{k l} \varepsilon_{k l}+\delta_{i j} \theta \Delta p \\
& =\Delta \sigma_{i j}^{\prime}+\delta_{i j} \theta \Delta p
\end{aligned}
$$

where $\sigma_{i j}^{\prime}$, is the effective stress tensor and $\lambda$ and $G$ are Lame's constants given by,

$$
\lambda=\frac{E}{2(1+\nu)}
$$

and

$$
G=\frac{E \nu}{(1+\nu)(1-2 \nu)},
$$

in which $E$ is Youngs Modulus, $\nu$ is Poisson's ratio and $G$ is Shear Modulus. Although, for $i, j=1,2,3$ equation (25) leads to 9 quantities of strain, the expression ultimately includes only 3 unknowns. These 3 unknowns are the 3 displacements $u_{1}, u_{2}$ and $u_{3}$ in the primary directions and the $\epsilon_{i j}$ involve spatial derivatives of these displacements.

Substitution of equation (25) into the force balance equation (24) gives rise to a set of three equations summarized in the following expression,

$$
\frac{\partial}{\partial x_{i}} \frac{\partial}{\partial x_{j}}\left(\lambda \varepsilon_{i j}+G \delta_{i j} \delta_{k l} \varepsilon_{k l}+\delta_{i j} \zeta \Delta p\right)_{\sigma, T}=0 .
$$

Equations (23), with the sum $\epsilon_{11}+\epsilon_{22}+\epsilon_{33}$ substituted for $\epsilon_{v}$ on the right hand side, and equation (27) together constitute a set of four coupled equations in 4 unknowns, namely, water pressure and the three displacements. These four equations fully describe the coupled process denoted by the partial differential, $\left(\partial M_{w} / \partial p\right)_{\sigma, T}$.

\section{$5.2(\partial p / \partial \sigma)_{M w, T}:$ Undrained loading}

In this case, as stresses are applied on the external boundaries of the system, the local stress field as well as the pore pressure will change throughout the flow domain subject to the constraint that 
the change in the volume of water is equal to the change in the volume of the voids. The set of four equations for this process will therefore entail 3 force balance equations which are the same as those of the transient flow process. However, the transient flow equation is to be replaced by an equation which represents the constraint that $\Delta V_{w}=\Delta V_{v}$. That is,

$$
\left(\varepsilon_{11}+\varepsilon_{22}+\varepsilon_{33}\right)_{M_{w}, T}=n \beta(\Delta p)_{M_{w}, T} .
$$

Equation (27), together with equation (28), therefore constitute a set of four coupled equations to consistently describe the differential $(\partial p / \partial \sigma)_{M_{w}, T}$.

\section{$5.3(\partial p / \partial T)_{\sigma, M_{w}}:$ Thermal pressurisation}

In this case, the heat equation influences the fluid flow equation by a thermally induced pressure change. At the same time, the fluid flow equation influences the heat equation through advective transport of heat by the flowing water. The effect of changing temperature at a given location is that the change in the volume of water due to thermal expansion has to be accommodated by an equivalent change in water pressure through thermal pressurisation. From equation (11), this equivalence may be expressed as,

$$
\frac{\left[n \beta_{T}-\alpha_{T}\right]}{\left[n \beta+\alpha_{I}\right]} \Delta T=\Delta p .
$$

This leads to a two-way coupling between the transient fluid flow equation and the transient heat equation as follows,

$$
\begin{gathered}
\frac{1}{\left[\alpha_{I}+n \beta\right]}\left(\nabla \cdot \frac{k}{\mu}[\rho g \nabla z+\nabla p]\right)_{\sigma, T}+\frac{\left[n \beta_{T}-\alpha_{T}\right]}{\left[n \beta+\alpha_{I}\right]} \\
\times\left(\frac{d T}{d t}\right)_{M_{w}, \sigma}=\left(\frac{\partial p}{\partial t}\right)_{\sigma, T}+\left(\frac{\partial p}{\partial t}\right)_{M_{w}, \sigma}, \quad(30) \\
-\nabla \cdot \rho c_{w} q+\nabla \cdot K_{T} \nabla T \\
=\left[\rho n c_{w}+(1-n) \rho_{s} c_{s}\right] \frac{d T}{d t},
\end{gathered}
$$

where $q$ is volumetric flux density of water, $c_{w}$ is the volumetric specific heat of water, $\rho_{s}$ is the density of solids, $c_{s}$ is the volumetric specific heat of the solids and $K_{T}$ is thermal conductivity of the watersolid mixture.

A subtle detail is worth noting here. Mathematically, one could multiply both sides of equation (30) by $\left[\alpha_{I}+n \beta\right]$, without changing the meaning. However, it is physically incorrect to do so, because the second term on the right hand side is an undrained process at constant mass content. Multiplying it by $\left[\alpha_{I}+n \beta\right]$ will violate mass conservation.

\section{Combining all three processes}

Now consider the general case, combining transient fluid flow with three dimensional deformation with heat flow and prescribed changes in external loads. This entails combining all the three partial derivatives detailed above. As a first step, consider undrained loading. Here, the change in water pressure at a given location is related to a change in external load according to, $\Delta p=B \Delta \sigma_{M}$, where $\Delta \sigma_{M}$ is the change in mean principal stress at that location within the flow domain, and $B$ is a material property given by equation (10). Therefore, to estimate $\Delta p$ at a given location at any time as the system evolves in time, it is necessary to know $\Delta \sigma_{M}$ at that location at the desired time as governed by the boundary conditions. If the patterns and magnitudes of boundary loads change continuously in time, the only way to obtain $\Delta \sigma_{M}$ at the given location and desired time would be to solve the coupled equations (27) and (28) repeatedly in time as the system evolves. However, a reasonable simplification is possible. If it is assumed that the patterns of boundary loads remain fixed in time, then, a single coupled solution of equations (27) and (28) will help establish a definite relationship between an incremental change in boundary loads and the change in $\Delta \sigma_{M}$ at any given location within the flow domain. In other words, the time derivative, $(\partial \sigma / \partial t)_{M_{w}, T}$ can be taken as an a priori known quantity. Accordingly, the simultaneous consideration of transient fluid flow, three dimensional deformation, and heat flow reduces to the following set of three coupled equations.

$$
\begin{aligned}
& \frac{1}{\left[\alpha_{I}+n \beta\right]}\left(\nabla \cdot \frac{k}{\mu} \nabla[\rho g \nabla z+\nabla p]\right)_{\sigma, T} \\
& +\frac{\left[n \beta_{T}-\alpha_{T}\right]}{\left[\zeta \alpha_{I}+n \beta\right]}\left(\frac{d T}{d t}\right)_{M_{w}, \sigma}+B\left(\frac{\partial \sigma}{\partial t}\right)_{M_{w}, T}=\frac{d p}{d t},
\end{aligned}
$$

$$
\begin{gathered}
\frac{\partial}{\partial x_{i}} \frac{\partial}{\partial x_{j}}\left(\lambda \varepsilon_{i j}+G \delta_{i j} \delta_{k l} \varepsilon_{k l}+\delta_{i j} \zeta \Delta p\right)_{\sigma, T}=0 . \\
-\nabla \cdot \rho c_{w} q+\nabla \cdot K_{T} \nabla T \\
=\left[\rho n c_{w}+(1-n) \rho_{s} c_{s}\right] \frac{d T}{d t} .
\end{gathered}
$$


Note that, in equation (32) the right hand side is the total time-derivative of water pressure, combining the response of pressure to all three causes.

If stress changes and strains in different directions are not of interest, the force balance equation (27) can be dispensed with. If the system is isothermal, the heat equation (31) can be disregarded, and the term involving $d T / d t$ can be dropped from equation (32). In such a case, the transient flow equation will account for transient fluid flow as well as effects of changing stresses such as earth tides or barometric tides. If external loads remain constant, then the term involving $B$ in equation (32) can also be dropped.

\section{A digression into energetics}

Our current state of scientific knowledge is such that earth systems in general and hydrogeological systems in particular are invariably treated within the framework of coupled differential equations. As seen from the ideas presented above, it is apparent that when one considers a several variable system, one assumes that each partial derivative is independent of the other, and that their individual effects can be superimposed. This assumption of superposition implies that the partial derivatives do not influence each other.

One may now ask if these coupled systems can be looked at in any other way, if for no reason other than curiosity. An alternate perspective, capable of providing insights is in fact possible. This involves looking at the system from the point of view of energy and work. The simplest case of a steady-state, isothermal groundwater flow system is described by Laplace's equation,

$$
\nabla \cdot \frac{k}{\mu}[\rho g \nabla z+\nabla p]=0 .
$$

It is well-known that equation (33) results from the minimization of the variational principle of the form,

$$
\Omega(\Phi)=\int_{V} K^{*}(\nabla \Phi)^{2} d V
$$

where $\Phi$ is a potential, defined as energy per unit mass of water (Hubbert 1940), and $K^{*}$ is a suitably defined conductivity. Physically, the integral on the right hand side of equation (34) represents the amount of energy expended as frictional heat per unit time, as water moves through the system. The minimization of equation (34) leading to equation (33) implies that the flow system organizes itself in such a way that energy is spent optimally in getting work done.

Now consider the transient isothermal flow problem, in which potentials change with time. Changing potentials in conjunction with hydraulic capacitance imply that some energy is retrievably stored in the system as potentials increase or decrease over the flow domain. Narasimhan (1999) showed that a variational principle can be formulated for the transient groundwater flow problem by postulating that, as the system evolves over a small interval of time $\delta t$, the sum of the energy expended in overcoming friction and the incremental energy retrievably stored is minimized. The relevant variational principle is,

$$
\begin{aligned}
\Omega(\Phi)= & \delta t \int_{V} K^{*}(\nabla \Phi)^{2} d V \\
& +\int_{V} c\left(\Phi\left(t_{0}+\delta t\right)-\Phi\left(t_{0}\right)\right)^{2} d V
\end{aligned}
$$

where $c$ is a suitably defined capacitance parameter. The second term on the right hand side of equation (35) denotes energy retrievably stored. Optimization of $\Omega(\Phi)$ implies that the system is evolving in an energetically favored direction.

If indeed the coupled system tends to evolve in an energetically favored direction, how is energy optimized among the different processes as it is partly dissipated and partly stored in various forms? Answering this question is fundamental to understanding how systems dictated by coupled processes may evolve with time. If certain processes are more favored than others, then the superposition of partial derivatives may not be justified. Yet, experimentally sorting out the partitioning of energy among various processes in coupled systems as the system evolves in time appears to be an insurmountable task. Under the circumstances, the only practical way of approaching these systems is to resort to the use of partial derivatives and the superposition of their effects.

\section{Acknowledgements}

This work was supported partly by the Agricultural Extension Service, through the Division of Natural Resources, University of California. Partial support was also provided by the Director, Office of Energy Research, Office of Basic Energy Sciences of the U.S. Department of Energy under Contract No. DE-AC03-76SF00098 through the Earth Sciences Division of Ernest Orlando Lawrence Berkeley National Laboratory. 


\section{References}

Biot M A 1941 General theory of three-dimensional consolidation; J. Appl. Phys. 12 155-164.

Hantush M S 1961 Drawdown around a partially penetrating well; J. Hyd. Div. Proc. Amer. Soc. Civil. Eng. July $83-98$.

Hubbert M K 1940 The theory of ground-water motion; J. Geol. 48 785-944.
Narasimhan T N 1999 Basic postulates of groundwater occurrence and movement; Proc. Indian Acad. Sci., Earth and Planet. Sci. 108 117-128.

Skempton A W 1954 The pore-pressure coefficients A and B; Geotechnique 4 143-147.

Terzaghi K 1923 Die Berechnung der Durchlässigkeitsziffer des Tones aus dem Verlauf der hydrodynamischen Spannungserscheinungen; [The calculation of permeability number of the clay out of the process of the hydrodynamic phenomenon tension.] Sitz. Akad. Wissen. Wien. Mathnaturw. Kl., Part Iia 32 125-138.

MS received 5 June 2005; revised 31 August 2005; accepted 2 September 2005 